\title{
A study of N-isopropyl acrylamide (NIPAM)-based polymer gel dosimeter by using Raman spectroscopy
}

\begin{abstract}
Polymer gel is a kind of radiation dosimetry system that has been applied widely in radiotherapy treatment. In this study, the samples of NIPAM-based polymer gel which posses a good potential as 3D radiation dosimetry are synthesized. The samples of polymer gel were irradiated up to $20 \mathrm{~Gy}$ by using gamma cell instrument with 60Co sources at a constant dose rate. Upon irradiation, then the chemical changes in polymer gel were characterized by using Raman spectroscopy. The polymerization was referred to an increment in Raman intensity at $815 \mathrm{~cm}-1$, assigned for C-C stretching mode of NIPAM polymer gel, as the dose increased. The consumptions of the co-monomers were referred to a decrement in Raman intensities at $1025 \mathrm{~cm}-1$ and $2353 \mathrm{~cm}-1$ assigned for $\mathrm{C}=\mathrm{C}$ stretching modes of NIPAM and BIS respectively, as the dose increased. Result shows that the amount of carbon single bonds increases while the amount of carbon covalent bonds decreases following irradiation.
\end{abstract}

Keyword: Polymer gel; Radiation dosimeter; Radiation therapy; Raman spectroscopy 